\title{
Cytomorphological Study of Oral Exfoliated Cells in Type II Diabetes Mellitus Patients
}

\author{
${ }^{1}$ Shruti Srinivasan, ${ }^{2} \mathrm{NS}$ Priya, ${ }^{3}$ Kavita Rao, ${ }^{4} \mathrm{HS}$ Umadevi, ${ }^{5} \mathrm{~T}$ Smitha, ${ }^{6} \mathrm{HS}$ Sheethal
}

\begin{abstract}
Introduction: Diabetes mellitus (DM) is one of the most common endocrine metabolic disorders and its prevalence has been increasing worldwide. It is a third leading cause of mortality and morbidity in the world. Diabetes affects several organs including the oral cavity. The oral complications of uncontrolled DM can include xerostomia, infection, poor healing, increased incidence and severity of caries, candidiasis, gingivitis, periodontal disease, periapical abscesses, and burning mouth syndrome. Early diagnosis of the DM is an important aspect of health care. Exfoliative cytology is considered a moderate, straightforward, and noninvasive diagnostic marker for DM. The purpose of this study is to demonstrate cytologic changes of the exfoliated oral mucosal cells of diabetic patients using hematoxylin and eosin (H\&E) staining technique to establish its role as diagnostic criteria.
\end{abstract}

Results: The frequency of micronuclei, enlarged nucleus, perinuclear halo, binucleation, cytoplasmic streaks, Candida, and inflammation were found to be statistically significant.

Conclusion: Clinical observations and results suggested morphologic and functional alterations in oral epithelial cells in diabetic patients, detectable by microscopic and cytomorphometric analysis using exfoliative cytology and can be used in diagnosis.

Clinical significance: The purpose of this study is to demonstrate cytologic changes of the exfoliated oral mucosal cells of diabetic patients using H\&E staining technique to establish its role as diagnostic criteria.

Keywords: Diabetes mellitus, Exfoliative cytology, Micronuclei. How to cite this article: Srinivasan S, Priya NS, Rao K, Umadevi HS, Smitha T, Sheethal HS. Cytomorphological Study of Oral Exfoliated Cells in Type II Diabetes Mellitus Patients. J Health Sci Res 2017;8(1):38-41.

Source of support: Nil

Conflict of interest: None

\section{INTRODUCTION}

Diabetes mellitus (DM) is a common endocrine metabolic disorder which brings in alterations in the equilibrium of

\footnotetext{
${ }^{1}$ Postgraduate Student, ${ }^{2,4,6}$ Reader, ${ }^{3}$ Professor and Head ${ }^{5}$ Professor

${ }^{1-6}$ Department of Oral Pathology, VS Dental College and Hospital, Bengaluru, Karnataka, India

Corresponding Author: Shruti Srinivasan, Postgraduate Student, Department of Oral Pathology, VS Dental College and Hospital, Bengaluru, Karnataka, India, Phone: +91-9900096081 e-mail: shrutiananthu@gmail.com
}

oral tissues and thereby alters the morphological features at cellular level.

Two major variants of DM are identified:

1. Type I DM/insulin-dependent DM, which accounts for 10 to $20 \%$ of all cases of primary diabetes.

2. Type II DM/noninsulin-dependent DM, which accounts for 80 to $90 \%$ of all cases of DM. ${ }^{1}$

Type II is more common than type I diabetes. The major classical findings of diabetes are polyphagia, polydipsia, polyuria, fatigue, and continuous weight loss. ${ }^{2}$ Frequent determination of glucose concentration in DM patients is an important tool in diabetes management, but requires repetitive venipuncture and finger lancing, which is painful and invasive. Hence, noninvasive or minimal invasive monitoring has been of particular interest. Oral exfoliative cytological study is once such noninvasive technique. ${ }^{3}$

Oral cytology is a relatively inexpensive, simple, and risk-free technique, i.e., well accepted by the patient with no contraindications. ${ }^{4}$ The morphologic and functional changes in oral mucosa can be studied at the cellular level by using exfoliative cytology which can help in the diagnosis, with better patient acceptability. ${ }^{3}$

Diabetes, being a metabolic disorder, has its effect on the metabolism of a cell and has its influence on several tissues in general and oral tissues in particular. There is decreased perfusion to the cell which affects the turnover, differentiation, and decrease in the energy-generating capacity of the cell. The cell and hence, the tissue shows certain changes thereof (Table 1).

The present study was undertaken to evaluate the cytomorphological changes like micronuclei, enlarged

Table 1: Diabetes and its effects on tissues ${ }^{1}$

\begin{tabular}{lc}
\hline Tissue changes & Cellular changes \\
\hline - Salivary dysfunction & Nuclear changes \\
- Taste dysfunction & - Enlarged nuclei \\
- Gingivitis & - Binucleation \\
- Periodontitis & - Karyorrhexis \\
- Dental caries & - Micronuclei \\
- Poor wound healing & - Perinuclear halo \\
- Bacterial infections & Cytoplasmic changes \\
- Candidiasis & - Increased cytoplasmic area \\
- Neuropathy & - Increased N:C ratio \\
- Angiopathy & - Granularity \\
& - Cytoplasmic vacuolation \\
\hline
\end{tabular}


nuclei, perinuclear halo, binucleation, cytoplasmic streak, presence of Candida, and inflammatory cells in exfoliated oral epithelial cells in diabetics.

\section{MATERIALS AND METHODS}

Smears from buccal mucosa of 100 known cases of DM patients (case group) and 100 nondiabetic healthy persons (control group) were obtained using wooden spatula. The scrapings were transferred to clean glass slides and spread thinly and uniformly and fixed in alcohol. The smears were then subjected for hematoxylin and eosin (H\&E) staining and were observed for cellular alterations like micronuclei, enlarged nuclei, perinuclear halo, binucleation, cytoplasmic streaks, presence of Candida, bacterial colonies, and inflammatory cells. Cells with clumping and overlapping were not included in the analysis. Subjects were selected considering the following inclusion and exclusion criteria (Table 2).

Results were tabulated and statistically analyzed using chi-square test and Fisher's exact test. p-value $\leq 0.05$ was considered to be statistically significant. The study was approved by the Ethical Committee of the institution.

\section{RESULTS}

In our study, smears from the buccal mucosa were evaluated using H\&E staining. In comparison between cases

Table 2: Inclusion and exclusion criteria

\begin{tabular}{ll}
\hline Inclusion criteria & Exclusion criteria \\
\hline - Normal buccal mucosa cells & - Smoker or alcoholic \\
of confirmed cases of Type II & patients \\
diabetic patients irrespective of & - Systemic diseases \\
the age, sex, and duration & - Pregnancy/menopause \\
- Patients with random glucose & state \\
level $>200 \mathrm{mg} / \mathrm{dL}$ & \\
\hline
\end{tabular}

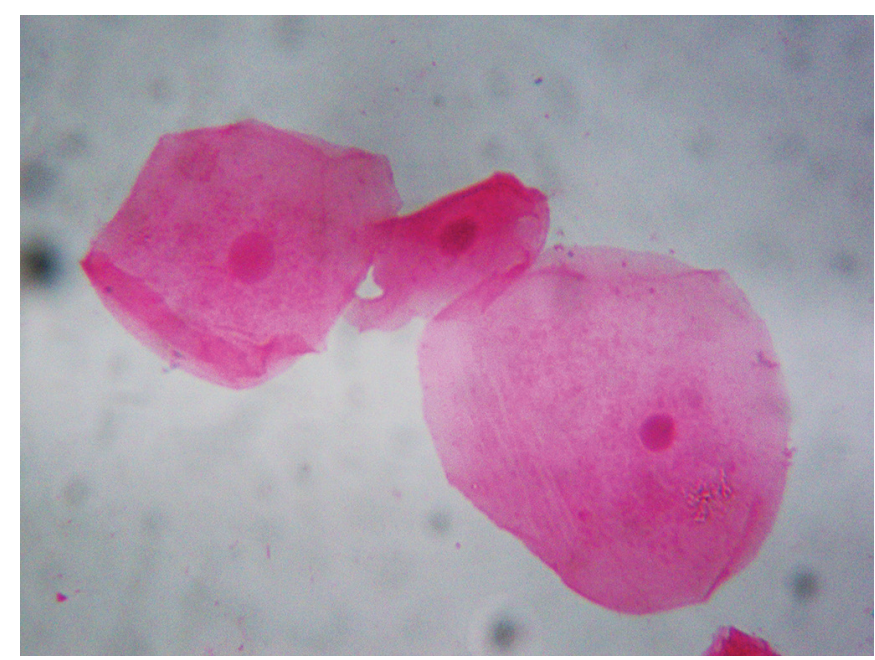

Fig. 2: Enlarged nuclei and control groups, among the case group, the frequency of micronuclei (Fig. 1), enlarged nuclei (Fig. 2), perinuclear halo, and binucleation (Fig. 3) were found to be statistically significant. Presence of candida and inflammation (Fig. 4), cytoplasmic streaks (Fig. 5) were assessed and compared statistically between cases and control groups and were found to be significant (Table 3, Graph 1).

An enlarged nucleus in few cases of diabetic group was found and was not statistically significant. Presence of bacterial colonies (Fig. 6) and inflammatory cytological atypia was noted in few cases of diabetic group.

Table 3: Comparison of variables between cases and controls

\begin{tabular}{lc}
\hline Variables & $p$-value \\
\hline Micronuclei & $<0.001$ \\
Enlarged nuclei & 0.25 \\
Perinuclear halo & 0.005 \\
Binucleation & $<0.001$ \\
Cytoplasmic streak & 0.009 \\
Candida & 0.008 \\
Inflammation & $<0.001$ \\
\hline
\end{tabular}

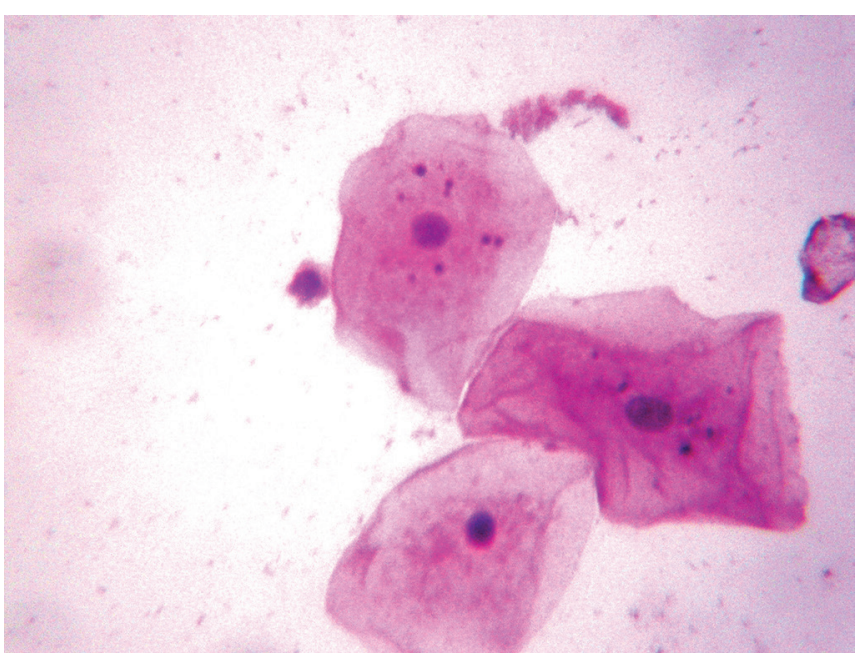

Fig. 1: Micronuclei

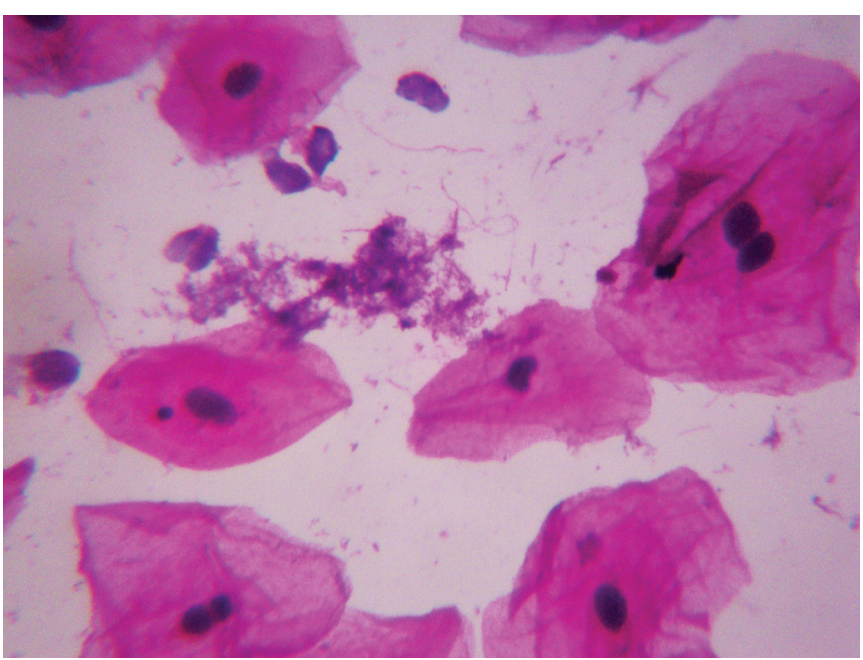

Fig. 3: Binucleation 


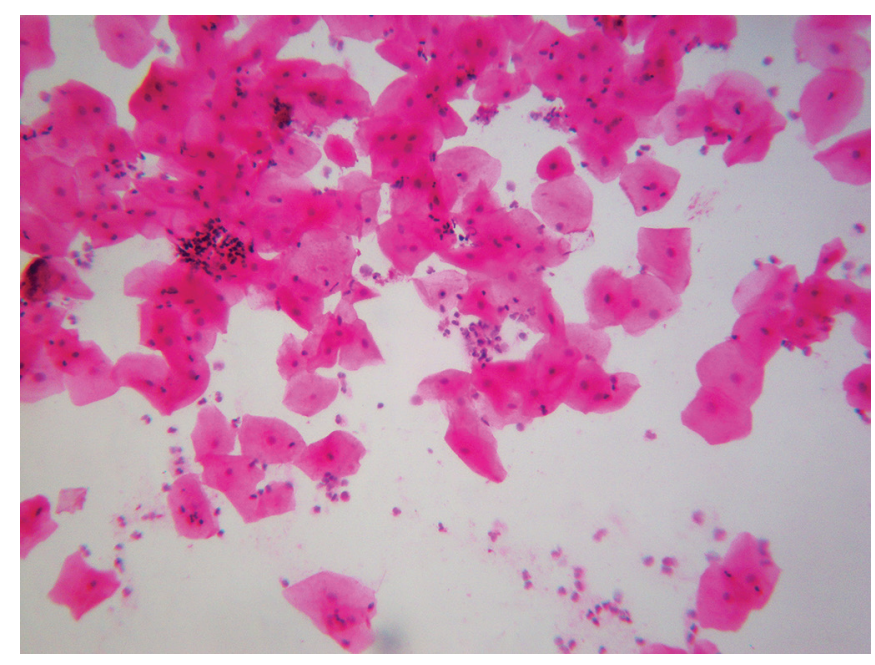

Fig. 4: Inflammatory cells

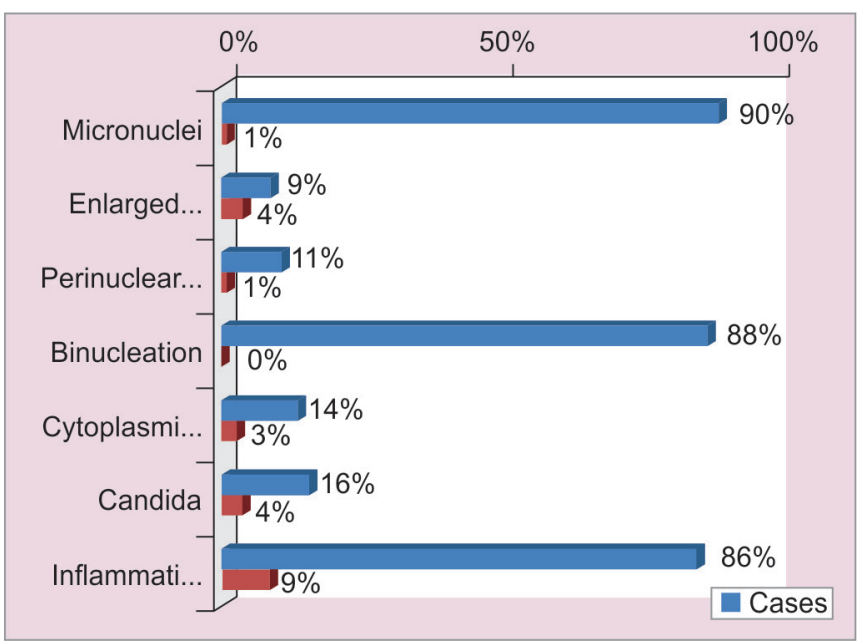

Graph 1: Graphical representation of variables between cases and controls

\section{DISCUSSION}

Epithelial cells undergo continuous renewal as a part of normal physiologic turnover. They move from the basal layer to the surface and are exfoliated. ${ }^{5}$ In DM, the cellular turnover is affected. Exfoliative cytology, being a noninvasive technique, allows simple and pain-free collection of intact cells from different layers within the epithelium for microscopic examination.

The term micronucleus (MN) test was suggested for the first time by Boller and Schmidt. It is defined as a microscopically visible round to oval cytoplasmic chromatin mass next to the nucleus. Micronucleus is an extra-nuclear cytoplasmic body which projects as a biomarker of chromosomal damage. ${ }^{6}$ Several studies have been conducted on micronuclei assay on the exfoliated cells of buccal mucosa of diabetic patients and have found that the frequency of micronuclei was increased in diabetic patients as compared to the normal individuals. Our results were consistent with the similar study conducted by Shashikala et al, ${ }^{6}$ Kashyap and Reddy,

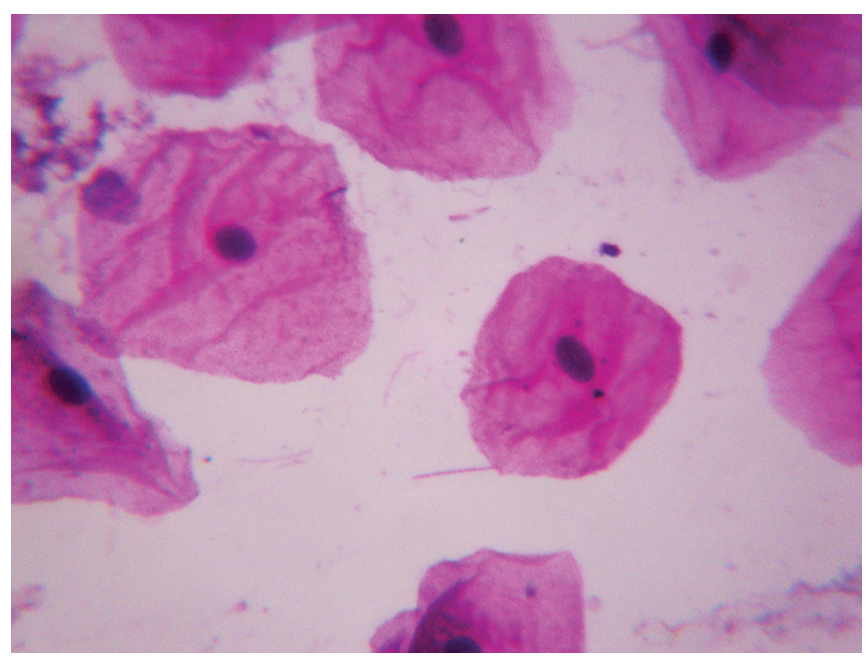

Fig. 5: Cytoplasmic streaks

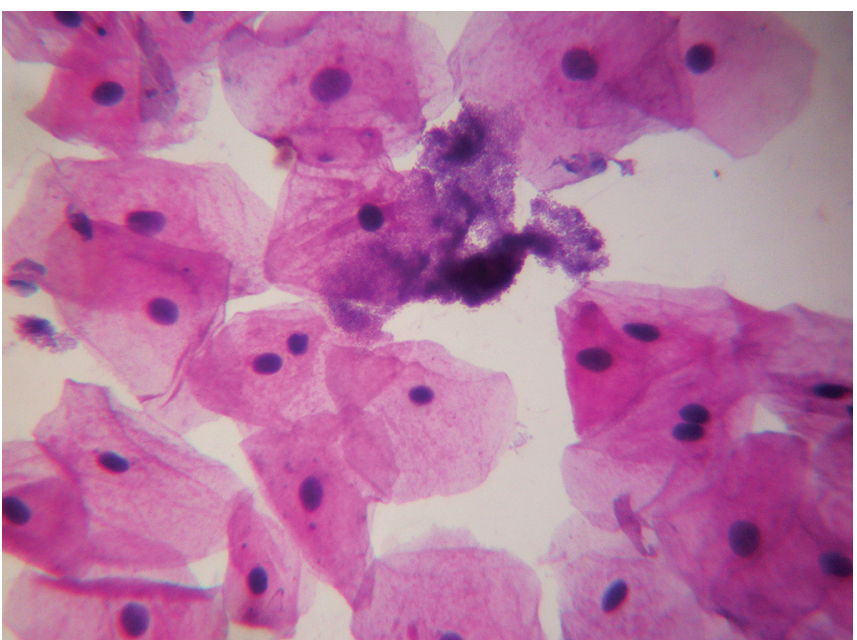

Fig. 6: Bacterial colonies

Holland et al, ${ }^{8}$ which showed increased prevalence of micronuclei in diabetic patients as compared to the normal individuals.

One of the parameters considered for the study was enlarged nuclei. In DM, there is decreased perfusion of the cells, which leads to decreased cellular turnover and keratinization. This delay in differentiation process of epithelium will lead to increase in the cells, which present a large nucleus as a primary characteristic. ${ }^{9}$ Delay in keratinization is attributed to glycogen changes. Sustained hyperglycemia causes greater accumulation of advanced glycation end products by abnormal glycation of proteins, lipids, and nucleic acids in the walls of large blood vessels as well as in basement membrane of the microvasculature. ${ }^{1}$

In a study conducted by Sonawane et $\mathrm{al}^{2}{ }^{2}$ Nandita et $\mathrm{al}^{1}$ using pulmonary alveolar proteinosis (PAP) stain showed that cells from diabetic patients exhibited an enlarged nucleus as compared to the cells from normal individuals. 
Increase in nuclear size might be an indicator of cellular ageing in diabetic patients. Decreased cellular turnover as a result of ischemia following atherosclerosis would result in more number of mature cells with large nuclei in the smear. Aging would also produce various morphologic alterations in cells in the form of pleomorphism, bilobed nuclei, cytoplasmic vacuolizations, etc. ${ }^{3}$ In our study, we were able to note such morphologic variations in epithelial cells from diabetic patients. A study conducted by Prasad et al, ${ }^{9}$ on 50 diabetic patients using PAP stain, showed that there was an increase in nuclear area in diabetic patients as compared to the normal individuals.

Oral candidiasis is seen more frequently in diabetic patients than in nondiabetics as they are more likely to have xerostomia and atrophic oral mucosa due to dehydration caused by the disease process as well as due to decrease in salivary flow rates. This is also associated with superadded infections like candidiasis, which can evoke a chronic inflammatory response in the oral mucous membrane. ${ }^{9}$ A study by Pallavan et $\mathrm{a}^{10}$ using PAP stain showed presence of severe candidal colonization in diabetic patients than the normal individuals.

A study conducted by Baban and Garib ${ }^{4}$ on 40 type II patients using PAP stain obtained from both buccal and gingival tissue showed that the study group showed bi and tri-nucleation and perinuclear halo. Cells from newly diagnosed diabetics showed irregular-shaped nuclei and increased inflammatory cells in the gingival smears.

A study conducted by Latti et $\mathrm{al}^{11}$ on 10 known diabetic patients and 10 control cases using H\&E, PAP, and periodic acid-schiff (PAS) showed that the study group exhibited binucleation, decreased cytoplasmic/nuclear ratio, nuclear enlargement, enucleation, and inflammation as compared to that of control group.

The scientific literature lacks sufficient relevant data, in relation to the use of H\&E stain in oral exfoliated cells in diabetes. Further detailed studies are required to ensure the validity of this stain. Moreover, H\&E stain is considered the primary and routine stain, inexpensive, widely used with easy interpretation of cellular details.

Other stains that can be used to study the exfoliated cells are PAP and PAS.

\section{CONCLUSION}

Diabetes mellitus is a silent metabolic killer with vast harmful effects on various organs. It affects the tissues at cellular level and cripples the normal functioning of the cell, which is manifested as various changes in the cell morphology. Our study is a basic study conducted on diabetic patients to observe the cytomorphological variations using exfoliative cytology as a diagnostic adjunct.

\section{SHORTCOMINGS}

- Since we have used H\&E stain, glycogen fails to stain with conventional hematoxylin solutions and only stains weakly, if at all, with eosin.

- Random glucose levels were considered for the study irrespective of the duration, since patient is diagnosed diabetic and irrespective of type of medications used.

\section{REFERENCES}

1. Nandita KP, Boaz K, Srikant N, Lewis AJ, Manaktala N. Oral epithelium in diabetics: a cytomorphometric correlation. Dent Hypotheses 2014;5(2):59-65.

2. Sonawane K, Jain S, Gupta I, Karthik BV, Singaraju S, Singaraju M. Cytomorphometric analysis of oral mucosa in diabetic patients in Bhopal region an in situ study. Int J Clin Dent Sci 2011;2(4):12-15.

3. HallikerimathS, Sapra G, Kale A,Malur PR. Cytomorphometric analysis and assessment of periodic acid Schiff positivity of exfoliated cells from apparently normal buccal mucosa of type 2 diabetic patients. Acta Cytolol 2011;55(2):197-202.

4. Baban MT, Garib BT. Cytological features of oral cytobrush smears in type ii diabetes mellitus patients. Tikrit J Dent Sci 2012;10:6-12.

5. Anuradha A, Sivapathasundharam B. Image analysis of normal exfoliated gingival cells. Indian J Dent Res 2007 Apr-Jun;18:63-66.

6. Shashikala R, Indira AP, Manjunath GS, Arathi Rao K, Akshatha BK. Role of micronucleus in oral exfoliative cytology. J Pharm Bioallied Sci 2015 Aug;7(Suppl 2);S409-S413.

7. Kashyap B, Reddy PS. Micronuclei assay of exfoliated oral buccal cells: means to assess the nuclear abnormalities in different diseases. J Cancer Res Ther 2012 Apr-Jun;8(2):184-191.

8. Holland N, Bolognesi C, Kirsch-Volders M, Bonassi S, Zeiger E, Knasmueller S, Fenech M. The micronucleus assay in human buccal cells as a tool for biomonitoring DNA damage: the HUMN project perspective on current status and knowledge gaps. Mutat Res 2008 Jul-Aug;659(1-2):93-108.

9. Prasad H, Ramesh V, Balamurali P. Morphologic and cytomorphometric analysis of exfoliated buccal mucosal cells in diabetes patients. J Cytol 2010 Oct;27(4):113-117.

10. Pallavan B, Ramesh V, Dhanasekaran BP, Oza N, Indu S, Govindarajan V. Comparison and correlation of candidal colonization in diabetic patients and normal individuals. J Diabetes Metab Disord 2014 Jun 4;13:66.

11. Latti BR, Birajdar SB, Latti RG. Periodic acid Schiff-diastase as a key in exfoliative cytology in diabetics: a pilot study. J Oral Maxillofac Pathol 2015 May-Aug;19(2):188-191. 